\title{
The Informatization Management Research of Large-Scale Pig Farm'S Healthy Aquaculture
}

\author{
Lifen Wang and Mingtao Ma* \\ Jilin Agricultural Science and Technology College Jilin 132101, Jilin,china \\ mmt800@126.com
}

Keywords: Informatization; Information management system; Healthy breeding

\begin{abstract}
This thesis pays attention to the study of actual situation of China's large-scale farms. Additionally, the information management system of pigs' health and cultivation has been developed to strengthen management level of healthy pigs, prevent epidemics, ensure the products quality of pigs, raise the scale, increase the output and input ratio and accelerate the development of aquaculture. The management system has mainly realized the pigs' breeding with assistance of computers, the management of disease, feeding and finance and other functions in the large-scale pig farms.
\end{abstract}

\section{Introduction}

With the continuous development of economy, China has more and more large-scale pig farms. The equipment is becoming more and more advanced, and the degree of automation is becoming higher and higher. Although China has the world's largest pig breeding and pork production, the gap on the rate of stock and carcass meat between China and those developed in the pig industry is still large. In general, the "scale" of China's pig farms is mainly reflected in the number of years of feeding and the number of annual columns, while the management of information and digitization is still low.

Informatization is the key to promote the development of animal husbandry, research on information technology in livestock and poultry production management, nutrition and feed, disease prevention and control in China has achieved certain results, but because of the rapid development of information technology, the current market sales management software is relatively old, affecting the pig raising management level

The scale of pig farms to improve the problems encountered in the development, must reform development, improve the breeding system and planning, improve the decision-making function of information and data, using a set of standardized management processes to regulate the breeding process of all business processes and business data, ensure the well documented, monitoring in place.In this study, in order to achieve the healthy breeding of pig pig farms as the core, as the object, the use of information technology (SQL Server 2012, Visual Studio.NET 2013, C\# development language), using standard farm feed, veterinary drugs and other inputs, strengthen the monitoring of rearing environment, design and development of pig breeding and health information management system to achieve, healthy breeding of pigs, and ensuring the quality and safety of pork products from the source, to accelerate the rapid and healthy development of the pig industry.

In order to guarantee the health of pigs, pork and related products, and the rapid development of pig breeding industry in Jilin province, it is necessary to use large-scale information management system for healthy farming, which is developed by a new information software, and to meet the market demand. 
System Requirement Analysis. The four management subsystems in the research include all aspects of health breeding for pig, including the collection and loading procedures of health standard CHINAGAP, the data of diagnosis and treatment of common diseases in pigs, all the respect of management in pig feeding and the edition of SQL Server 2012 database by using financial management data. After the compilation of subroutine information, the author uses specialized computer programming language Visual Studio and.NET 2013 development environment and C\# development language subsystem to make the Subsystem editor link into a complete information-based managerial system of pig breeding.

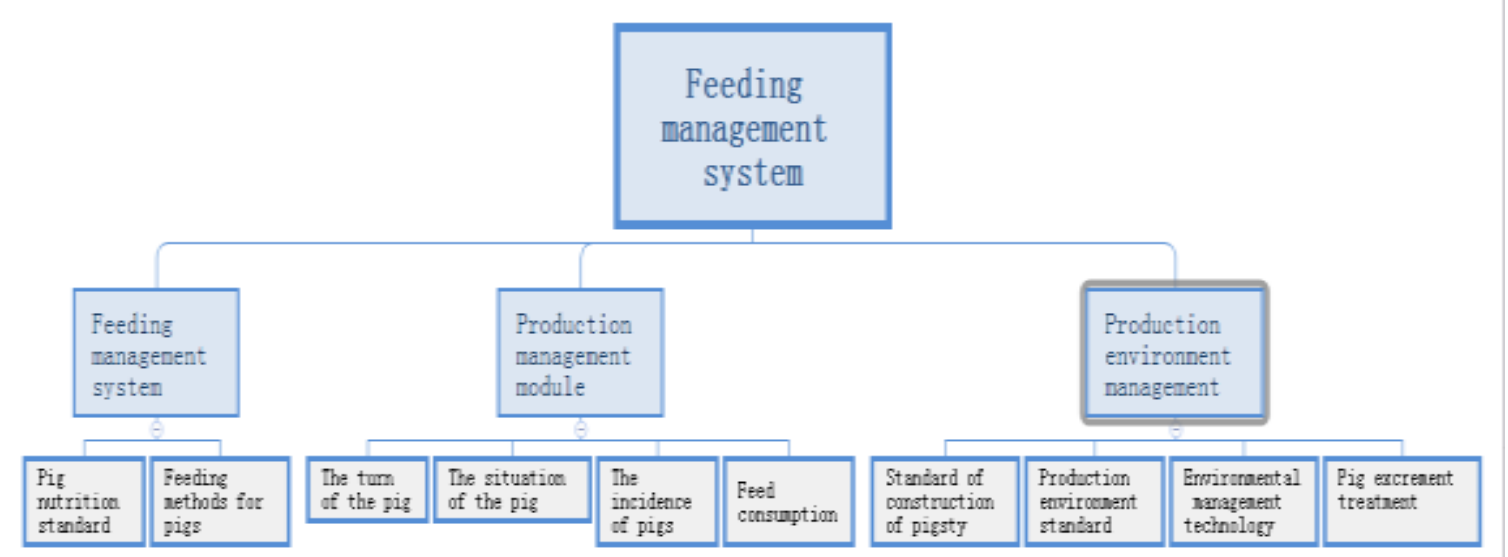

Figure. 2-1 Disease management system

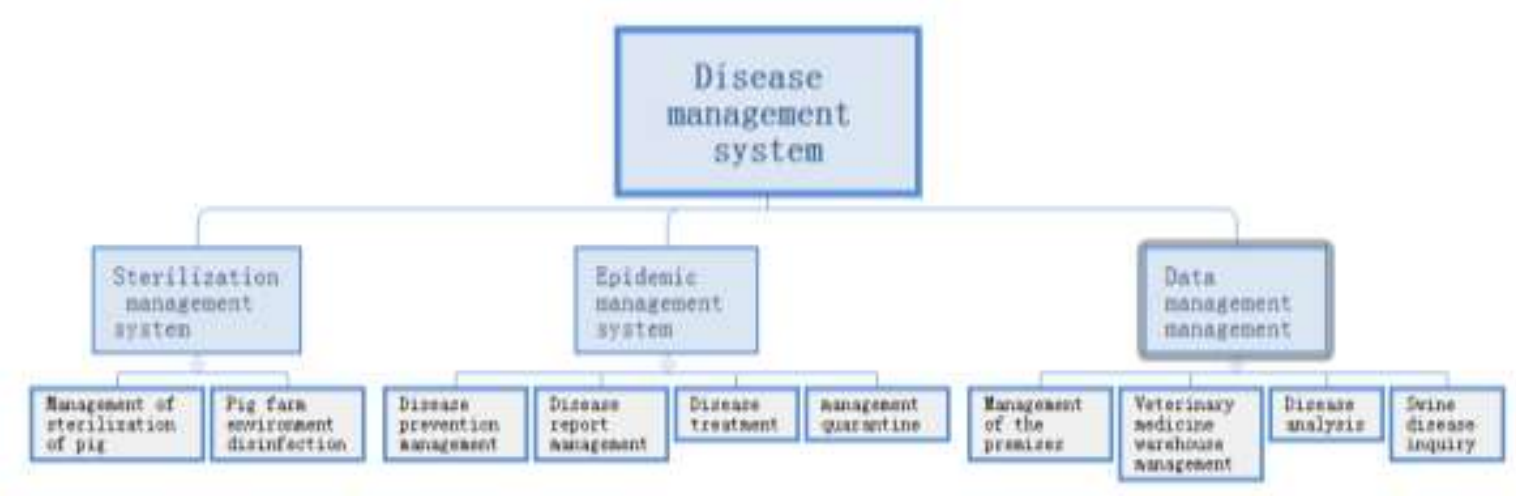

Figure. $\quad 2-2$ Feeding management system 


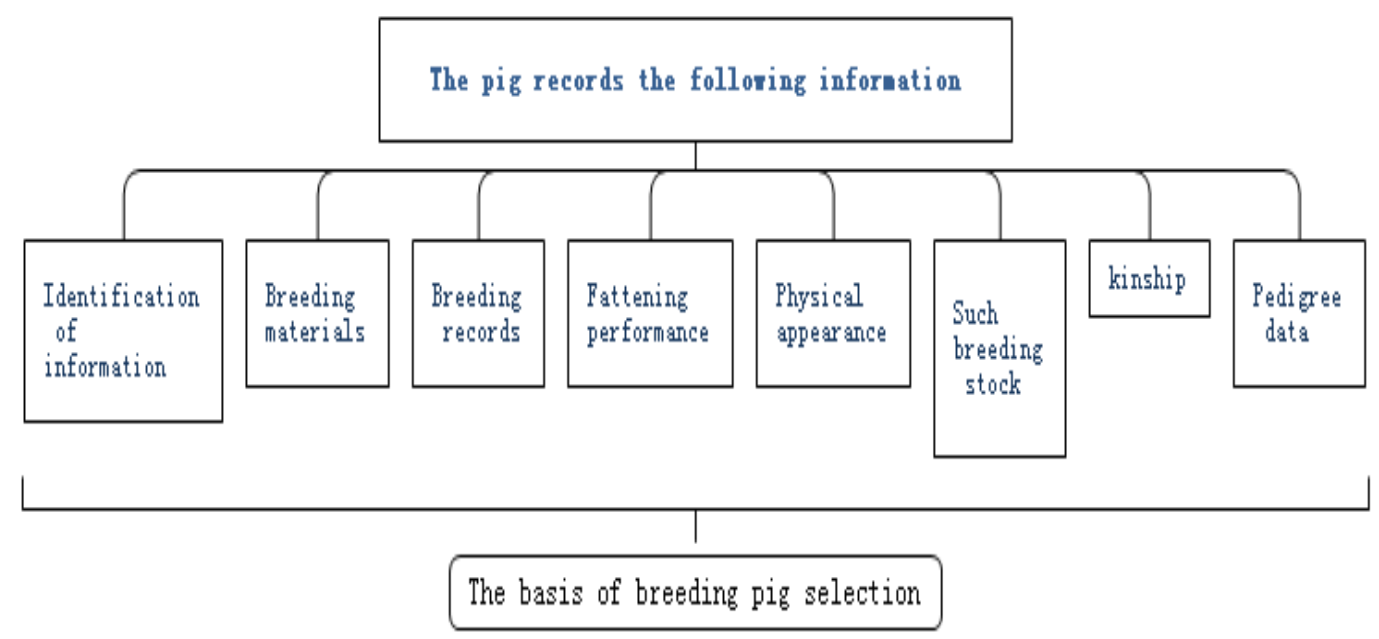

Figure. 2-3 Computer-aided breeding system

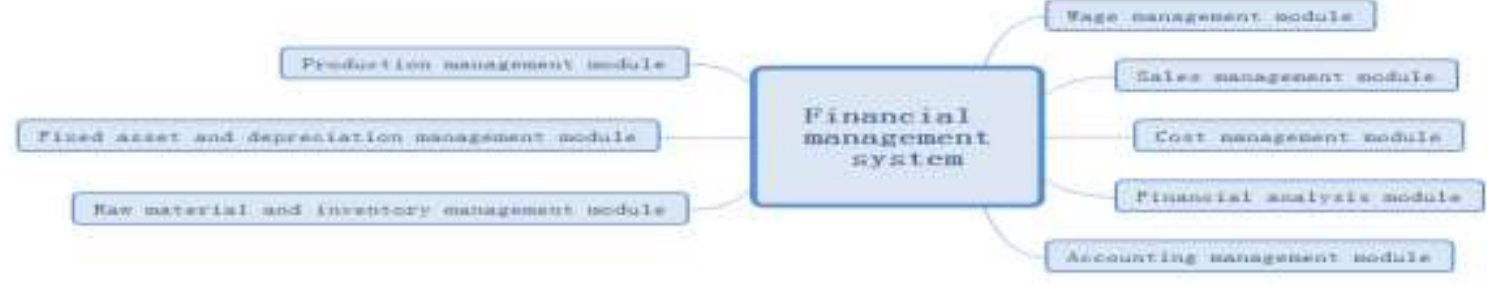

Figure. 2-4 Financial management module

\section{Design and Implementation}

\section{System Design}

System design based on B/S architecture, WEB ASP.NET Internet service platform research and development technology, represents the future of management software architecture is the future development direction, support the "cloud computing". Support asynchronous data exchange, work site and server support asynchronous data communication, more user-friendly operation, data statistics and more rapid and smooth uninterrupted data support. The Internet, centralized data service, $24 \times 7$ online services, making data handy. Support cross platform access, whether it is personal computer, PDA or intelligent mobile phone, realize seamless data in TCP/IP protocol communication environment interface and interaction, operating on the terminal device, the data "trigger"

\section{Design of Technical Scheme}

This system use the B/S structure, this kind of structure support cross the platform, system and browser, it can make the user experience effect and benefit better. Because of the B/S system can make the user use independent, it can access control the character.

This system use $\mathrm{C}$ language for the people, first, set up a system operating environment, then Deploying installing. The working environment:

operating: Window7

exploitation environment: Visual Studio.NET 2013 
development tool: C\#.net

application server: SQL Server 2012

Brower English: IE8.0

\section{Conclusion}

Through the use of information management construction methods, the pig farm in the intelligent management and artificial management, can be based on their own conditions for the rational design of the system and frame design. At the same time, by drawing on the experience and process of successful pig farms at home and abroad, the optimal solution is adopted to realize the intelligent management of the quality of pig breeding. The system makes full use of modern information management system, realize the function, network technology and intelligent data analysis and data query, through the analysis of continuous improvement and system, promote the intelligent management of Jilin province pig breeding.

This paper mainly introduces the development background of the system, technical scheme, overall design, detailed design and so on. The content of farm management, breeding management, breeding management, drug management and other business applications are analyzed, expounds the realization of the system function.

\section{Acknowledgements}

*Foundation item: Science and Technology Research Project supported by Education Department of Jilin Province during the Thirteen Five-Year Plan period (Grant No.2016200)

\section{References}

[1] Yin Chengcheng, Shandong province pig industry development strategy research, Shandong Agricultural University, 2014, (05): 41-45

[2] Design and Research on Distributed Real-time Monitoring System of Nongying Camp and Scale Livestock and Poultry Farms, Jiangsu University, 2009,42-45

[3] Hutong based on intelligent pig breeding management system design and research, agricultural network information 2015,59-62

[4] WU You-hua, WANG Jing, CHENG Wen-chao.Nowing the rural recreation households should pay attention to the prevention of poultry disease [J]. Scientific breeding. 2014 (05)

[5] GAO Wan-lin, Research on Integrated Information Management System of Pig Farm (English), Journal of Agricultural Engineering, 2015: 230-236 